"This is the peer reviewed version of the following article: [Al-Ganmi A.H., Perry L., Gholizadeh L. \& Alotaibi A.M. (2016) Cardiovascular medication adherence among patients with cardiac disease: a systematic review. Journal of Advanced Nursing ]which has been published in final form at [ http://dx.doi.org/10.1111/jan.13062 ]. This article may be used for non-commercial purposes in accordance with Wiley Terms and Conditions for Self-Archiving." 


\title{
Cardiovascular medication adherence among patients with cardiac disease: a systematic review
}

\author{
Ali Hussein Al-Ganmi BSc MSc RN, Lin Perry MSc PhD RN, Leila Gholizadeh BSc \\ MSc PhD RN, Abdulellah Modhi Alotaibi MSc RN
}

\begin{abstract}
Aims: To critically appraise and synthesise the best available evidence on the effectiveness of interventions suitable for delivery by nurses, designed to enhance cardiac patients' adherence to their prescribed medications.
\end{abstract}

Background: Cardiac medications have significant health benefits for patients with heart disease, but patients' adherence to prescribed medications remains suboptimal.

Design: A systematic quantitative review of intervention effects.

Data Sources: We conducted systematic searches for English-language, peer-reviewed randomised controlled trial publications via Medline, EMBASE, CINAHL, the Cochrane Library, ProQuest, Web of Science and Google Scholar published between January 2004 and December 2014.

Review methods. According to pre-determined inclusion and exclusion criteria, eligible studies were identified and data extracted using a predefined form. Of 1,962 identified papers; 14 studies met the study inclusion criteria, were assessed for risk of bias using the Cochrane Collaboration tool; and included in the review.

Results: Study findings were presented descriptively; due to the heterogeneity of studies meta-analysis was not possible. Included papers described interventions categorised as: 1) multifaceted, and 2) behavioural and educational, comprising: 2a) text message and mail message, 2b) telephone calls, 2c) motivational interviewing and 2d) nurse-led counselling and education.

Conclusions: Substantial heterogeneity limited the robustness of conclusions, but this review indicated that motivational interviewing, education, and phone or text messaging appeared promising as means to enhance cardiac medication adherence. Future research should 
integrate multifaceted interventions that target individual behaviour change to enhance adherence to cardiovascular medications, to build on the beneficial outcomes indicated by this review.

\section{Summary Statements}

\section{Why is this research or review needed?}

- Non-adherence to cardiovascular medicines is associated with greater risk of hospitalisation and mortality.

- Various studies examine the effectiveness of medication adherence interventions, but the evidence is inconsistent and unclear.

- Nurses play a key role in educating and supporting patients with their medication, but evidence is lacking for effective nurse-delivered interventions to promote medication adherence for cardiac patients.

\section{What are the key findings?}

- Multifaceted interventions that target individual behaviour change appear to offer most promising methods to enhance adherence to cardiovascular medications.

- Current evidence indicates that the components for multifaceted interventions most likely to sustain adherence to cardiac medication-taking include motivational interviewing, education and phone or text messaging.

\section{How should the findings be used to influence policy/ practice/research?}

- Findings provide evidence to support clinicians' choice of interventions designed to enhance medication adherence among patients with cardiac disease delivered by nurses. 
- Rigorous methods are required for future studies to test the effectiveness of nurse-led adherence interventions, taking into account study design, sampling, choice of effective and feasible adherence measures, ways to accommodate interventions within routine daily practice and longer term sustainability of outcomes.

- Policy-makers should consider the opportunities offered by nurse-led medication adherence interventions, to enhance the contribution of nurses and thereby optimise the benefits to patients of their prescribed medications.

Keywords: cardiovascular medication, adherence, cardiac disease, systematic review, text message, motivational interviewing, education, nursing, nurse-led intervention.

\section{Introduction}

Medication non-adherence is a complex problem that poses an enormous health and economic burden. It is more common among older age population and patients who need multiple medications for their chronic conditions (Menditto et al. 2015). It has been estimated that inadequate adherence to treatment among patients with chronic diseases affects up to $50 \%$ of patients (Lee et al. 2006, Wilke et al. 2011). Non-adherence is a multifactorial phenomenon, affected by socio-economic status, health systems, disease states, pharmacological therapies and patient beliefs (Sabaté 2003). Supporting long-term adherence to medicines is an essential component of patient management and requires effective interventions to help achieve sustained medication-taking. This systematic review therefore aimed to identify effective interventions used by nurses to improve cardiac patients' adherence to cardiac medications.

\section{Background}

Cardiovascular disease (CVD) remains the leading cause of mortality among men and women and is responsible for one third of all deaths worldwide (World Health Organisation 2004). In developing countries, coronary heart disease (CHD) accounts for more than 4.5 million 
deaths per annum (Okrainec et al. 2004). Similar to international trends, CVD is the single greatest cause of death in Australia, accounting for the deaths of 11,733 males and 9,780 females in 2011(Australian Institute of Health and Welfare 2014). Although CHD is incurable, disease progress can be significantly slowed by cardio-protective medicines and lifestyle changes. Cardio-protective medicines are the primary therapy for CHD, but adherence to these medications is suboptimal, resulting in insufficient control of disease symptoms and increased risk of future cardiovascular events, rehospitalisation and death (Baroletti\& Dell'Orfano 2010). The World Health Organisation define adherence as "the extent to which a person's behaviour (taking medications, following a recommended diet and/or executing life-style changes) corresponds with the agreed recommendations of a health care provider" (Sabaté 2003). Adherence to medication recommendations is necessary to receive the full benefits of the medications. Medication non-adherence has been defined as "taking less than $80 \%$ of prescribed doses and can also include taking too many doses", and it is associated with an increased risk of poor health, adverse clinical events, and death (Nieuwlaat et al. 2014). It is estimated that up to $50 \%$ of patients with CHD in high income countries do not take their medications as prescribed (Laba et al. 2013). Poor medication adherence has been linked to recurrent cardiac events and adverse patient outcomes (Poluzzi et al. 2011, Nieuwlaat et al. 2014).

The prevalence of patients in the aging Australian population who are non-adherent to cardiovascular medications has been reported to range from $14 \%$ to $43 \%$, posing a serious barrier to secondary prevention (McKenzie et al. 2015). Thus, long term medication adherence in Australia remains unsatisfactory, with the situation changing only very slowly (Simons et al. 2011). Poor medication adherence rates undermine the translation of the benefits of well-established evidence-based cardiovascular medicines into practice, reducing the effectiveness of secondary prevention therapies (Haynes et al. 2005). It is crucial that 
adherence to cardiovascular medicines is optimised to improve disease symptoms and prevent the onset of further serious cardiac events (van Dalem et al. 2012).

The effectiveness of a wide variety of interventions intended to enhance medication adherence in patients with CHD has been trialled. A number of diverse and complex behavioural, educational, and combined intervention approaches and outcomes measures have emerged; however, the effectiveness of these interventions needs to be carefully evaluated due to the diverse methodologies used in the studies. It is important that healthcare professionals are aware of effective practical strategies and have the necessary skills to translate these interventions to outpatient healthcare settings. The purpose of this systematic review was to identify and synthesise the best available evidence on the effectiveness of interventions within the nursing scope of practice, designed to enhance cardiac patients' adherence to cardio-protective medications.

\section{The review}

\section{Aims}

Many and varied approaches have been trialled with the intention of enhancing medication adherence, but what interventions suitable for delivery by nurses are effective at improving the adherence of cardiac patients to their prescribed cardio-protective medications?

\section{Design}

A systematic quantitative review of intervention effects was conducted according to the principles and processes of the Cochrane Handbook for Systematic Reviews of Interventions (Higgins\& Green 2008). GRADE was used for grading the quality of evidence provided by findings (EPOC Resources for review authors 2013). The PRISMA statement (Moher et al. 2009) was used to guide reporting of the review. 


\section{Search methods}

A systematic literature search was conducted using six electronic databases: Medline, EMBASE, CINAHL, the Cochrane Library, ProQuest, and Web of Science. These databases and Google Scholar were searched for articles published in English between January 2004 and December 2014. The reference lists of all selected articles including review articles were searched for additional studies. The keywords used in the search strategy were based on the 'PICOS' framework (Table 1).

Studies were included if they were primary research reported the results of un-confounded evaluation of interventions suitable for delivery by nurses to increase medication adherence for patients with CHD. The following inclusion criteria were applied:

1) Study design was a RCT, clinical trial, or controlled clinical trial that examined the effectiveness of an intervention to increase adherence to medications among patient with cardiac disease, used for secondary prevention or treatment of cardiac disease in which an intervention group was compared to a control group who received standard care or a clearly justified comparison group;

2) The population of interest comprised male and female adults ( $\geq 18$ years old) with a diagnosis of a cardiac disease;

3) The intervention strategy was suitable (within the scope of practice) for delivery by nurses and had either a primary or secondary aim to increase the adherence to medication of patient with cardiac disease;

4) Patients were followed for at least 6 months;

5) Medication was self-administered, that is, was not administered by a health care professional or carer, and measured by any method e.g.: pill count, electronic monitoring, refill or prescription records, or self-reported data. 
Studies were excluded if they targeted patients with heart valve disease or post-cardiac surgeries; were written in a language other than English; included non-cardiac disease patients; tested interventions that required delivery by a non-nursing healthcare professional, e.g. pharmacist; were conducted in inpatient settings; and had less than six months follow-up, because cardiovascular medications typically require long-term adherence.

\section{Search outcome}

In total, search strategies identified 1,962 citations of potential relevance. Initial screening of study titles and abstracts revealed that more than $95 \%$ of the retrieved studies did not meet the review inclusion criteria, leaving 94 papers for further evaluation. The full texts of these papers were then reviewed and 14 studies were retained for assessment. The flow of studies through the selection process is summarised in Figure 1.

\section{Quality appraisal}

The quality of included studies was appraised by three authors (AA, LP \& LG). Risk of bias assessment is presented in the supplementary online Table S1 using the Cochrane Collaboration tool for assessing the risk of bias (Higgins et al. 2011). Risk of bias was assessed independently by the authors, and discrepancies were resolved by discussion (Figure 2).

\section{Data abstraction}

Data were extracted and analysed by three authors (AA, LP \& LG) using a predefined form. After quality appraisal of these studies, 14 were retained for the review. Details of data extracted are available in the supplementary online Table S1. 


\section{Synthesis}

Multiple sources of heterogeneity (interventions, adherence measures, and outcomes) were observed across the included studies; formal meta-analysis was therefore not appropriate. The heterogeneity was explored qualitatively by comparing the characteristics of included studies. Studies were grouped according to the main components of the interventions (see the supplementary online Table S2).

\section{Results}

These fourteen included studies contained data on 4,548 patients with cardiac disease. These studies were summarised based on the country of origin, participants' cardiac disease diagnostic group, and the practice setting of interventions (Table 2). None of these studies detailed or considered the potential effect of socio-demographic, literacy or economic characteristics on intervention outcomes. The median follow-up time was one year, ranging from six months to 24 months. Most studies achieved their endpoint outcomes at three to nine months. Interventions varied, with single, combined, and multifaceted component parts. Interventions and study characteristics are detailed in Table S2.

\section{Risk of bias assessment}

Risk of bias was assessed using the Cochrane Collaboration tool (Higgins et al. 2011) for selection, performance, attrition, detection, reporting and systematic bias. All trials provided information about adequate sequence generation, ten studies described the measures used to blind outcome assessors from group allocation. Four studies avoided performance bias (Jiang et al. 2007, Ogedegbe et al. 2008, Beune et al. 2014, Leiva et al. 2014) by providing information about adequate blinding of participants and personal. Ten studies reported participants lost to follow up; six trials provided study protocols and reported the methods of 
outcomes assessment (medication adherence). The remaining studies reported all outcomes but without study protocols (Schroeder et al. 2005, Jiang et al. 2007, Ogedegbe et al. 2008, Smith et al. 2008, Guirado et al. 2011, Kripalani et al. 2012, Rinfret et al. 2013, Leiva et al. 2014) (see Figure 2).

GRADE was used to rate the quality of evidence for medication adherence. Scores ranged -1 or +1 and were summed to produce overall scores $(4=$ high, $3=$ moderate, $2=$ low and $1=$ very low) based on the risk of bias, design, inconsistency, indirectness and imprecision in their scores (EPOC Resources for review authors 2013). Most studies were at low or unclear risk of bias and the quality of evidence was rated moderate for the outcomes (Figure 2). Hence, study results should be interpreted with caution.

\section{Medication adherence measurement}

Methods for measuring and monitoring medication regimen adherence varied in these trials. Six studies each measured adherence by self-report (Jiang et al. 2007, Guirado et al. 2011, Hacihasanoğlu\& Goözuöm 2011, Nieuwkerk et al. 2012, Beune et al. 2014, Ma et al. 2014) and by pharmacy refill electronic data (Smith et al. 2008, Rinfret et al. 2013, Ho et al. 2014, Leiva et al. 2014, Wald et al. 2014). The Medication Event Monitoring System (MEMS) pill bottle caps was used in one study (Schroeder et al. 2005), and two studies applied both selfreport and MEMS (Ogedegbe et al. 2008, Kripalani et al. 2012).

\section{Types of intervention}

Interventions were categorised according to their prominent components and included: 1) multifaceted and 2) behavioural and educational interventions. The latter comprised: $2 \mathrm{a}$ ) text message and mail message, 2b) telephone calls, 2c) motivational interviewing, and 2d) nurseled counselling and education. The complex nature of some interventions made them difficult to categorise, but this was based on the main component of intervention. Three studies 
examined text message (TM) and/or mail message interventions (Smith et al. 2008, Kripalani et al. 2012, Wald et al. 2014); two studies tested multifaceted intervention strategies (Ho et al. 2014, Leiva et al. 2014); two studies investigated the effect of structured telephone calls (Hacihasanoğlu\& Goözuöm 2011, Rinfret et al. 2013); and two studies utilised the motivational interviewing approach (Ogedegbe et al. 2008, Ma et al. 2014). Five interventions were classified as nurse-led counselling and education (Schroeder et al. 2005, Jiang et al. 2007, Guirado et al. 2011, Nieuwkerk et al. 2012, Beune et al. 2014). The heterogeneity of interventions, measurement tools and methods precluded meta-analysis of baseline and post-intervention rates of adherence for the reviewed studies.

\section{Multifaceted interventions}

The effectiveness of multifaceted interventions for enhancing medication adherence was described and evaluated by two studies. Ho et al. (2014) utilised a four stage multifaceted intervention that entailed: medication reconciliation and tailoring, education about medications, collaborative care, and two types of scheduled voice messaging (educational and medication refill reminder calls). Similarly, Leiva et al. (2014) evaluated a multifaceted intervention incorporating motivational interviewing, pillbox reminders, family support, blood pressure measurements and antihypertensive reminder forms, and simplification of dosing regimens in patients with hypertension. Ho et al. (2014) found that adherence rate improved at 12 months follow-up by $89.3 \%$ in the intervention arm compared with $73.9 \%$ with usual care for four classes of medications. On the other hand, Leiva et al. (2014) found no significant between groups differences in antihypertensive adherence at 12 months $(51.4 \%$ vs. 50.8\%) in intervention and control groups, respectively (Table S2). The Ho et al. (2014) study was high quality; however, Leiva et al. (2014) did not employ a blinding process and delivery of the intervention by nurses varied according to their characteristics and the 
methods of delivery, possibly resulting in overestimation of the intervention effect (Tables S2). Overall, this type of intervention approach appeared likely to increase adherence to medications after hospital discharge post ACS and was costed at $\$ 360$ per patient per year (Ho et al. 2014).

\section{Text and mail message interventions}

Three studies assessed the effectiveness of text message (TM) (Wald et al. 2014) and mail message reminders (Smith et al. 2008, Kripalani et al. 2012). In the Wald et al. (2014) study, participants in the intervention group received automatically generated daily TM reminders which questioned patients whether they had taken their blood pressure and/or lipid lowering medications; whether the message had reminded them to take it; if they had forgotten or whether they had simply not taken it. This study showed $16 \%$ improvement in medication adherence $(95 \%$ CI 7\%-24\%, $\mathrm{p}<0.001)$ at 6 months follow-up, and a statistically significant difference between groups of patients who had stopped medication completely and those who continued to take $<80 \%$ of the prescribed regimen (Table S2). However, participants' high adherence rates at baseline and unclear randomisation and blinding procedures may have resulted in under or overestimation of intervention effects (Tables S1).

Two studies applied a less individual approach, one mailed graphical postcards focusing on refill and other important reminders to patients with CHD (Kripalani et al. 2012), another focused on improving cognitive aspects of medication adherence by sending two letters to patients and to primary care providers describing the importance of beta-blockade (Smith et al. 2008). Kripalani et al. (2012) showed a non-significant difference in improvements in adherence between groups (32.9\% vs. 32.9\% respectively) whereas the Smith et al. (2008) study improved adherence rates among patients in the intervention group by $17 \%$, increasing the days covered to $80 \%$ in this group (relative risk $=1.17 ; 95 \% \mathrm{CI}=1.02-1.29 ; \mathrm{p}=0.04$ ). The 
quality of Smith et al. (2008) study was good in terms of randomisation methods, intervention and strategies used for applying the intervention and follow-up. By contrast, graphical mailed refill reminders failed to improve medication adherence, attributed to quality issues with randomisation and blinding processes (Table S1). Overall, the TM and mail message approaches appeared potentially effective and feasible strategies as reinforcement for taking medication and improving medication adherence.

\section{Telephone call interventions}

Two studies (Hacihasanoğlu\& Goözuöm 2011, Rinfret et al. 2013) examined the use of structured phone calls with interactive components to improve medication adherence. Hacihasanoğlu\& Goözuöm (2011) randomly allocated patients with hypertension to three groups to receive a 6-month nurse-based medication educational intervention alone, educational intervention plus home monitoring for medication adherence, or a control group. Both intervention groups received monthly follow-up phone call interviews providing them with information about hypertension. Similarly, in the Rinfret et al. (2013) study, patients with dual antiplatelet therapy (DAT) $(n=150)$ were randomised to either nurse phone calls within one week and then at one month, six months and nine months to assess adherence, reinforce optional drug compliance and discuss the factors affecting adherence or to a control group (Table S2). Both studies showed a significant increase in medication adherence using different measures. At 10 months follow up, there was a significant increase in regular medication intake ratios after education in groups $\mathrm{A}$ and $\mathrm{B}(80 \%, 85 \%$, respectively, $\mathrm{p}=$ 0.001) but not in the control group (42\%, p> 0.05) (Hacihasanoğlu\& Goözuöm 2011). Combined education (group B) was shown to have a more positive effect on adherence selfefficacy than education alone (group A) and no intervention (control group) $(72.27$ (SD= 5.27); $71.10(\mathrm{SD}=6.42) ; 56.85(\mathrm{SD}=6.10)$ respectively, $\mathrm{F}=83.131 ; \mathrm{p}=0.001)$ 
(Hacihasanoğlu\& Goözuöm 2011). The Rinfret et al. (2013) study showed that participants in both groups had high adherence to antiplatelet drugs at 12 months, with $99.2 \%$ (ranging from $97.5 \%$ to $100 \%$ ) of the intervention group taking aspirin compared to $90.2 \%$ (ranging from $84.2 \%$ to $95.4 \%$ ) of the control group; clopidogrel, $99.3 \%$ (ranging from $97.5 \%$ to $100 \%$ ) in the intervention group vs. $91.5 \%(85.1-96.0 \%) \%$ in the control group, $(\mathrm{p}<0.0001)$. However, the study results may have been biased by the lack of blinding of patients and intervention providers, although it was impractical in this study (Table S1).

\section{Motivational interviewing strategies}

Motivational interviewing has been used as an approach to increase adherence to medication in cardiac patients. Ogedegbe et al. (2008) conducted a randomised controlled trial in two community-based primary care practices in the US, evaluating the efficacy and effects of practice-based motivational interview (MINT) counselling on medication adherence and blood pressure in 190 African American patients with hypertension. Based on intention-totreat analysis using mixed-effects regression, the MINT group achieved a higher MEMS adherence rate at 12 months follow-up compared to the control group (57\% vs. $43 \%$ respectively, $p<0.05)$, with an absolute between-group difference of $14 \%$ (95\% CI, -0.2 to 27\%). The MINT group received behavioural counselling about medication adherence for 30-40 minutes at three, six, nine, and 12 months, which led to steady maintenance of medication adherence over 12 months, while adherence rates declined overtime in the control group. Similarly, Ma et al. (2014) applied MINT counselling, based on social cognitive theory, to 120 patients with hypertension from two community health centres in China. This intervention entailed strategies to promote adherence to behaviour changes, summarising the pros and cons of proposed behaviour changes, setting realistic and specific goals for behaviour modification and prompting patients to follow plans for behaviour change (Table 
S2). Adherence to medication was improved in the MINT counselling group compared to the control group at six months follow-up (29.72 ( $\mathrm{SD}=3.46)$ vs. $25.30(\mathrm{SD}=3.11)$ respectively, $(t=0.039, p=0.034)$. Mean scores for medication adherence were increased within-groups with mean difference between baseline and six months for intervention group of $(23.25$ (SD= $3.02) ; 29.72(\mathrm{SD}=3.46)$ respectively, $(t=0.039, P=0.034)$ and $(22.13(\mathrm{SD}=2.89) ; 25.30$ $(\mathrm{SD}=3.11)(t=0.039, P=0.061)($ Table $\mathrm{S} 2)$. This form of MINT was theory-based, and shown to be effective using accepted valid measures for adherence assessment over longer duration of follow-up.

\section{Nurse-led counselling and education}

Five studies intended to improve medication adherence by using behavioural interventions and education through nurse-led counselling. Four studies demonstrated no or little evidence of effect (Schroeder et al. 2005, Jiang et al. 2007, Guirado et al. 2011, Beune et al. 2014). These results may be attributed in part at least to feature of the research methods, such as self-selected populations with high adherence levels at baseline (Schroeder et al. 2005) and randomisation and blinding processes bias (Jiang et al. 2007, Guirado et al. 2011, Beune et al. 2014) (Table S1). Nieuwkerk et al. (2012) revealed that adherence to lipid lowering medication increased from $95 \%$ to $100 \%$ in the intervention group and from $90 \%$ to $95 \%$ in the control group. At 18 months follow up, the intervention group had higher adherence to statin therapy than the control group $(9.39(\mathrm{SD}=0.15)$ vs $8.86(\mathrm{SD}=0.15))$ respectively, with an absolute difference between groups of $0.53(0.02-1.05),(\mathrm{r}=-0.36, \mathrm{P}<0.01)$ (Table S2). With 201 patients on statin therapy randomised to receive nurse-led multifactorial cardiovascular risk-factor counselling or standard care, significant outcomes were attributed to the more extensive personal contact with the nurse practitioner as well as risk-factor counselling in the intervention group compared to the control group. Overall, most studies demonstrated 
no improvement in outcomes from nurse-led behavioural interventions (Schroeder et al. 2005, Jiang et al. 2007, Guirado et al. 2011, Beune et al. 2014), whilst Nieuwkerk et al. (2012) study appeared to offer an opportunity to improve medication adherence. However, taking into consideration the baseline difference between groups, these study findings should be interpreted with caution.

\section{Discussion}

This review of interventions to enhance adherence to medications in cardiovascular care highlighted the varying effectiveness of approaches trialled, but also the varying methods of evaluation. The evidence of effectiveness presented for these interventions was inconsistent, due at least in part to the different cardiac disease populations and adherence measurement methods used in these studies. Results were not materially affected by age, sex or smoking (Wald et al. 2014) but the impact of socioeconomic status could not be determined; however, examination of the effect of these factors went beyond the review research question (Jiang et al. 2007).

Motivational interviewing, either alone or combined with another adherence approach such as phone or text message education and reminders, appeared the most promising behavioural intervention for improving medication adherence, with potential for wide application across patients with different forms of cardiac disease. Interventions that utilised motivational interviewing (MINT) strategies were successful at maintaining medication adherence over time among hypertensive patients (Ogedegbe et al. 2008). The results support those of previous studies, demonstrating that MINT may be a useful approach for addressing medication adherence (DiIorio et al. 2008) because it enhance patient readiness to change, increase their confidence in their ability to overcome barriers, and increase self-motivation to achieve desired outcomes (Rollnick\& Miller 1995). This approach entailed setting realistic 
and specific goals for behaviour modification and prompted patients to follow their plan for behaviour change. This can be achieved when trained nurses integrate adherence behaviour into patients' daily routines and reinforce the positive effect of MINT by follow-up using phone calls, text messaging, or mails as a mean of multifactorial intervention.

Multifaceted interventions also demonstrated statistically significant improvement in medication adherence (Ho et al. 2014). Study findings were consistent with other successful multifaceted interventional studies, which have included medication review with a specific focus on regimen simplification (Bernsten et al. 2001), individualised patient education combined with medication reminders (Hawe\& Higgins 1990), or a dose administration aid (Lee et al. 2006). These results also accorded with a study (Edworthy et al. 2007) that found significant improvement in adherence for both beta-blocking and lipid-lowering agents with counselling by nurses and pharmacists along with video, printed material, and phone followup. Multifaceted interventions have broadly demonstrated promising results, but make it difficult to draw conclusions in favour of any particular combination of interventions or intensity because of the heterogeneity and complexity of interventions, compounded by multiple adherence measures (Topinková et al. 2012), and drug classes (van Eijken et al. 2003). The cost of these interventions are also an important consideration for roll-out, this could not be determined as interventions were not described in sufficient detail.

A common element of many medication adherence interventions was education. However, the methods of delivering education differed and outcomes were inconsistent. Effective nurse-based medication educational interventions included six monthly face to face education sessions (Hacihasanoğlu\& Goözuöm 2011) about the important of regular medication taking, medication efficacy, possible side effects, and the importance of follow-up visits. Medication education was also successfully combined with automatic voice message reminders at one week and one month after hospital discharge for 12 months (Ho et al. 2014). By contrast, 
written medication educational materials delivered by a nurse combined with three structured counselling sessions was not associated with significant improvement in medication adherence (Guirado et al. 2011, Beune et al. 2014).

Comparisons across assessments of adherence was also difficult. The medication event monitoring system (MEMS), one of the most reliable objective assessment methods was expensive and not readily available for some dose forms (Remington et al. 2007, van den Boogaard et al. 2011). Subjective self-reporting measures are commonly used as they are relatively simple and less expensive; a number are well-validated and have been strongly correlated with objective measures of adherence in different populations (Nguyen et al. 2014).

For text and mail message interventions, studies showed similar improvements in medication adherence, at 16\% (Wald et al. 2014) and 17\% (Smith et al. 2008), respectively. These findings are consistent with recent RCTs reporting that bidirectional text messages resulted in statistically significant improvement in anti-retroviral treatment adherence among patients with Human Immunodeficiency Virus (Lester et al. 2010) and with hypertension (Márquez Contreras et al. 2005). Similarly, phone call interventions significantly improved adherence self-efficacy to antihypertensive medications at 10 months follow-up when combined with health promotion theory-based medication education and behavioural modification targeting patients' life styles (Hacihasanoğlu\& Goözuöm 2011). Cutrona et al. (2010)'s review concluded that phone calls both by trained lay people and by a nurse yielded significant improvements in cardiovascular adherence. Likewise, tailored telephone call nursing interventions reduced the time commitment, the cost for the care provider, provider costs, and improved medication adherence for patients with chronic diseases (Bosworth et al. 2009). However, a review by Mansoor et al. (2013) found that informational interventions had little or no impact on improving medication adherence. This could be due to how well health care 
providers delivered the interventions, the patient groups, study design and differences in the relative contribution of each element to the intervention. For example, information supplied passively to the patient may not be adequate, and the additional element of requiring a response from the patient may be what was responsible for significant change. All in all, using technology in the form of phone message intervention provided by nurses appeared feasible, cost-effective and likely to be an effective tool to improve medication adherence in resource-limited settings.

The results of nurse-led interventions were mixed although one study (Nieuwkerk et al. 2012) was successful in increasing adherence rates for lipid-lowering medications by enhancing patients' knowledge through structured counselling sessions. A similar intervention was shown to be beneficial in patients with hypertension (Logan et al. 1983). Nurse-led interventions have also failed to show positive effects on medication adherence in other populations. For example Clarke et al. (2002) found no significant between-group differences in mean change scores of medicine taking after 12 months of a nurse-led diabetes management program. However, overall, the evidence is not adequate, with current studies limited by short follow-up, small sample size, and inconsistent adherence measures.

To date, of nurse-delivered interventions to improve adherence to cardiovascular medications, multifaceted interventions appear to offer the best opportunities to optimise medication adherence, with component behavioural interventions in the form of motivational interviewing, educational content, text and/or phone messaging showing the greatest success.

This review has some limitations. All papers reviewed were from English-language sources and published since 2004; study results may not represent less contemporary non English publications. The wide variety of medication adherence measurement methods utilised in these studies made it difficult to detect changes in adherence to medications, although, most 
studies did use validated self-report questionnaires. Finally, the lack of concealment of randomisation allocation, blinding, self-reporting bias, and high rate of participant dropout in some studies could compromise the integrity of the study data. Hence, for each study, we examined potential biases that might explain differences among studies.

\section{Conclusion}

The prevalence of CVD is rising rapidly worldwide alongside emerging new and complex medication regimens which challenge both patients and healthcare providers (Hauptman 2008). Of the interventions intended to improve adherence to cardiovascular medications tested by studies in this review, multi-component interventions, tailored to address the patients' health behaviours, appeared to offer most promise. Combined interventions need to be detailed and employ multiple approaches such as motivational interviewing and education that target the desired behaviour change, and reinforcement of these behaviours such as with phone or text message strategies. However, whilst this review provides pointers for promising intervention approaches available to nurses, further studies are required to develop and test ways to accommodate these promising interventions within daily practice. It is imperative that interventions chosen are theory-based, and evaluated in robust trials to demonstrate effects on clinical outcomes, feasibility in usual practice settings, and sustainability. There is clearly a future role for technology in automating management of, for example, text and mail messaging. Healthcare providers in primary and secondary health settings should maximise the health benefits offered by medications by adopting those strategies shown to be effective at enhancing patients' adherence to their medications. Researchers should clearly justify and specify methodologies to generate a cumulative body of knowledge that can be used to inform clinical practice. Further investigation of factors affecting long-term medication adherence is warranted to enable better targeting of interventions. 
In summary, this review flags the enormous potential for future research and nursing practice development to significantly contribute to the care and outcomes of cardiac patients through optimising the benefits offered by medication schedules. Review findings indicate promising outcomes, but also highlight the current paucity of high quality research and knowledge deficits in this field.

\section{References}

Australian Institute of Health and Welfare (2014) Leading causes of death in Australia. In Australia's health series no. 14. Cat. no. AUS 178. Canberra: AlHW. Retrieved from http:// www.aihw.gov.au/workarea/downloadasset.aspx?id=60129548150.

Baroletti, S. \& Dell'Orfano, H. (2010) Medication Adherence in Cardiovascular Disease. Circulation, 121(12), 1455-1458.

Bernsten, C., Björkman, I., Caramona, M., Crealey, G., Frøkjær, B., Grundberger, E., Gustafsson, T., Henman, M., Herborg, H. \& Hughes, C. (2001) Improving the well-being of elderly patients via community pharmacy-based provision of pharmaceutical care. Drugs \& Aging, 18(1), 6377.

Beune, E.J.A.J., Moll van Charante, E.P., Beem, L., Mohrs, J., Agyemang, C.O., Ogedegbe, G. \& Haafkens, J.A. (2014) Culturally Adapted Hypertension Education (CAHE) to Improve Blood Pressure Control and Treatment Adherence in Patients of African Origin with Uncontrolled Hypertension: Cluster-Randomized Trial. PLoS ONE, 9(3), e90103.

Bosworth, H.B., Olsen, M.K., Dudley, T., Orr, M., Goldstein, M.K., Datta, S.K., McCant, F., Gentry, P., Simel, D.L. \& Oddone, E.Z. (2009) Patient education and provider decision support to control blood pressure in primary care: A cluster randomized trial. The American Heart Journal, 157(3), 450-456.

Clarke, J., Crawford, A. \& Nash, D.B. (2002) Evaluation of a comprehensive diabetes disease management program: progress in the struggle for sustained behavior change. Disease Management, 5(2), 77-86.

Cutrona, S.L., Choudhry, N.K., Fischer, M.A., Servi, A., Liberman, J.N., Brennan, T. \& Shrank, W.H. (2010) Modes of delivery for interventions to improve cardiovascular medication adherence: Review. The American Journal of Managed Care, 16(12), 929-942.

Dilorio, C., McCarty, F., Resnicow, K., Holstad, M.M., Soet, J., Yeager, K., Sharma, S.M., Morisky, D.E. \& Lundberg, B. (2008) Using motivational interviewing to promote adherence to antiretroviral medications: A randomized controlled study. AIDS Care, 20(3), 273-283. 
Edworthy, S.M., Baptie, B., Galvin, D., Brant, R.F., Churchill-Smith, T., Manyari, D. \& Belenkie, I. (2007) Effects of an enhanced secondary prevention program for patients with heart disease: A prospective randomized trial. Canadian Journal of Cardiology, 23(13), 1066-1072.

EPOC Resources for review authors (2013) Effective Practice and Organisaton of Care (EPOC). Cochrane Effective Practice and Organisation of Care.

Guirado, E.A., Ribera, E.P., Huergo, V.P., Borras, J.M. \& Group, A. (2011) Knowledge and adherence to antihypertensive therapy in primary care: results of a randomized trial. Gaceta Sanitaria, 25(1), 62-67.

Hacihasanoğlu, R. \& Goözuöm, S. (2011) The effect of patient education and home monitoring on medication compliance, hypertension management, healthy lifestyle behaviours and BMI in a primary health care setting. Journal of Clinical Nursing, 20(5/6), 692-705.

Hauptman, P. (2008) Medication adherence in heart failure. Heart Failure Reviews, 13(1), 99-106.

Hawe, P. \& Higgins, G. (1990) Can medication education improve the drug compliance of the elderly? Evaluation of an in hospital program. Patient Education and Counseling, 16(2), 151-160.

Haynes, R., Yao, X., Degani, A., Kripalani, S., Garg, A. \& McDonald, H. (2005) Interventions for enhancing medication adherence. The Cochrane Library.

Higgins, J.P. \& Green, S. (2008) Cochrane handbook for systematic reviews of interventions, Wiley Online Library.

Higgins, J.P.T., Altman, D.G., Gøtzsche, P.C., Jüni, P., Moher, D., Oxman, A.D., Savović, J., Schulz, K.F., Weeks, L. \& Sterne, J.A.C. (2011) The Cochrane Collaboration's tool for assessing risk of bias in randomised trials. British Medical Journal, 343.

Ho, P.M., Lambert-Kerzner, A., Carey, E.P., Fahdi, I.E., Bryson, C.L., Melnyk, S.D., Bosworth, H.B., Radcliff, T., Davis, R. \& Mun, H. (2014) Multifaceted intervention to improve medication adherence and secondary prevention measures after acute coronary syndrome hospital discharge: a randomized clinical trial. JAMA Internal Medicine, 174(2), 186-193.

Jiang, X., Sit, J.W. \& Wong, T.K.S. (2007) A nurse-led cardiac rehabilitation programme improves health behaviours and cardiac physiological risk parameters: evidence from Chengdu, China. Journal of Clinical Nursing, 16(10), 1886-1897.

Kripalani, S., Schmotzer, B. \& Jacobson, T.A. (2012) Improving Medication Adherence through Graphically Enhanced Interventions in Coronary Heart Disease (IMAGE-CHD): a randomized controlled trial. Journal Of General Internal Medicine, 27(12), 1609-1617.

Laba, T.-L., Bleasel, J., Brien, J.-a., Cass, A., Howard, K., Peiris, D., Redfern, J., Salam, A., Usherwood, T. \& Jan, S. (2013) Strategies to improve adherence to medications for cardiovascular diseases in socioeconomically disadvantaged populations: a systematic review. International Journal of Cardiology, 167(6), 2430-2440.

Lee, J.K., Grace, K.A. \& Taylor, A.J. (2006) Effect of a pharmacy care program on medication adherence and persistence, blood pressure, and low-density lipoprotein cholesterol: a randomized controlled trial. The Journal of the American Medical Association, 296(21), 25632571.

Leiva, A., Aguiló, A., Fajó-Pascual, M., Moreno, L., Martín, M.C., Garcia, E.M., Duro, R.E., Serra, F., Dagosto, P., Iglesias-Iglesias, A.A., Company, R.M., Yañez, A., Llobera, J. \& On behalf of The Adherence, G. (2014) Efficacy of a brief multifactorial adherence-based intervention in reducing blood pressure: a randomized clinical trial. Patient Preference and Adherence, $\mathbf{8}$, 1683-1690.

Lester, R.T., Ritvo, P., Mills, E.J., Kariri, A., Karanja, S., Chung, M.H., Jack, W., Habyarimana, J., Sadatsafavi, M., Najafzadeh, M., Marra, C.A., Estambale, B., Ngugi, E., Ball, T.B., Thabane, L., Gelmon, L.J., Kimani, J., Ackers, M. \& Plummer, F.A. (2010) Effects of a mobile phone short message service on antiretroviral treatment adherence in Kenya (WelTel Kenya1): a randomised trial. The Lancet, 376(9755), 1838-45. 
Logan, A.G., Milne, B., Flanagan, P. \& Haynes, R. (1983) Clinical effectiveness and cost-effectiveness of monitoring blood pressure of hypertensive employees at work. Hypertension, 5(6), 828836.

Ma, C., Zhou, Y., Zhou, W. \& Huang, C. (2014) Evaluation of the effect of motivational interviewing counselling on hypertension care. Patient Education and Counseling, 95(2), 231-237.

Mansoor, S.M., Krass, I. \& Aslani, P. (2013) Multiprofessional interventions to improve patient adherence to cardiovascular medications. Journal of Cardiovascular Pharmacology And Therapeutics, 18(1), 19-30.

Márquez Contreras, E., Vegazo García, O., Martel Claros, N., Gil Guillén, V., de la Figuera von Wichmann, M., Casado Martínez, J.J. \& Fernández, R. (2005) Efficacy of telephone and mail intervention in patient compliance with antihypertensive drugs in hypertension. ETECUMHTA study. Blood Pressure, 14(3), 151-158.

McKenzie, S., McLaughlin, D., Clark, J. \& Doi, S.R. (2015) The Burden of Non-Adherence to Cardiovascular Medications Among the Aging Population in Australia: A Meta-Analysis. Drugs \& Aging, 32(3), 217-225.

Menditto, E., Guerriero, F., Orlando, V., Crola, C., Di Somma, C., Illario, M., Morisky, D.E. \& Colao, A. (2015) Self-Assessment of Adherence to Medication: A Case Study in Campania Region Community-Dwelling Population. Journal of Aging Research, 2015, 682503.

Moher, D., Liberati, A., Tetzlaff, J. \& Altman, D.G. (2009) Preferred reporting items for systematic reviews and meta-analyses: the PRISMA statement. Annals Of Internal Medicine, 151(4), 264.

Nguyen, T.M.U., Caze, A.L. \& Cottrell, N. (2014) What are validated self-report adherence scales really measuring?: a systematic review. British Journal of Clinical Pharmacology, 77(3), 427445.

Nieuwkerk, P.T., Nierman, M.C., Vissers, M.N., Locadia, M., Greggers-Peusch, P., Knape, L.P.M., Kastelein, J.J.P., Sprangers, M.A.G., de Haes, H.C. \& Stroes, E.S.G. (2012) Intervention to Improve Adherence to Lipid-Lowering Medication and Lipid-Levels in Patients With an Increased Cardiovascular Risk. The American Journal of Cardiology, 110(5), 666-672.

Nieuwlaat, Wilczynski N, Navarro T, Hobson N, Jeffery R, Keepanasseril A, Agoritsas T, Mistry N, lorio A, Jack S, Sivaramalingam B, Iserman E, Mustafa RA, Jedraszewski D, Cotoi C \& B, H.R. (2014) Interventions for enhancing medication adherence. Cochrane Database of Systematic Reviews, (11).

Ogedegbe, G., Chaplin, W., Schoenthaler, A., Statman, D., Berger, D., Richardson, T., Phillips, E., Spencer, J. \& Allegrante, J.P. (2008) A practice-based trial of motivational interviewing and adherence in hypertensive African Americans. American Journal of Hypertension, 21(10), 1137-1143.

Okrainec, K., Banerjee, D.K. \& Eisenberg, M.J. (2004) Coronary artery disease in the developing world. American Heart Journal, 148(1), 7-15.

Poluzzi, E., Piccinni, C., Carta, P., Puccini, A., Lanzoni, M., Motola, D., Vaccheri, A., De Ponti, F. \& Montanaro, N. (2011) Cardiovascular events in statin recipients: impact of adherence to treatment in a 3-year record linkage study. European Journal of Clinical Pharmacology, 67(4), 407-414.

Remington, G., Kwon, J., Collins, A., Laporte, D., Mann, S. \& Christensen, B. (2007) The use of electronic monitoring $\left(\mathrm{MEMS}^{\circledR}\right)$ to evaluate antipsychotic compliance in outpatients with schizophrenia. Schizophrenia Research, 90(1-3), 229-237.

Rinfret, S., Rodés-Cabau, J., Bagur, R., Déry, J.-P., Dorais, M., Larose, É., Barbeau, G., Gleeton, O., Nguyen, C.-M., Noël, B., Proulx, G., Roy, L., Taillon, I., De Larochellière, R., Bertrand, O.F. \& Investigators, f.t.E.-I. (2013) Telephone contact to improve adherence to dual antiplatelet therapy after drug-eluting stent implantation. Heart, 99(8), 562-569.

Rollnick, S. \& Miller, W.R. (1995) What is motivational interviewing? Behavioural and Cognitive Psychotherapy, 23(04), 325-334. 
Sabaté, E. (2003) Adherence to long-term therapies: evidence for action, Geneva, Switzerland: World Health Organisation.

Schroeder, K., Fahey, T., Hollinghurst, S. \& Peters, T.J. (2005) Nurse-led adherence support in hypertension: a randomized controlled trial. Family Practice, 22(2), 144-151.

Simons, L.A., Ortiz, M. \& Calcino, G. (2011) Long term persistence with statin therapy: Experience in Australia 2006-2010. Australian Family Physician, 40(5), 319-22.

Smith, D.H., Kramer, J.M., Perrin, N., Platt, R., Roblin, D.W., Lane, K., Goodman, M., Nelson, W.W., Yang, X. \& Soumerai, S.B. (2008) A randomized trial of direct-to-patient communication to enhance adherence to $\beta$-blocker therapy following myocardial infarction. Archives of Internal Medicine, 168(5), 477-483.

Topinková, E., Baeyens, J.P., Michel, J.-P. \& Lang, P.-O. (2012) Evidence-Based Strategies for the Optimization of Pharmacotherapy in Older People. Drugs \& Aging, 29(6), 477-494.

van Dalem, J., Krass, I. \& Aslani, P. (2012) Interventions promoting adherence to cardiovascular medicines. International Journal of Clinical Pharmacy, 34(2), 295-311.

van den Boogaard, J., Lyimo, R.A., Boeree, M.J., Kibiki, G.S. \& Aarnoutse, R.E. (2011) Electronic monitoring of treatment adherence and validation of alternative adherence measures in tuberculosis patients: a pilot study. Control electrónico del cumplimiento terapéutico de pacientes con tuberculosis y validación de medidas alternativas de cumplimiento: estudio piloto., 89(9), 632-639.

van Eijken, M., Tsang, S., Wensing, M., de Smet, P.A.G.M. \& Grol, R.P.T.M. (2003) Interventions to improve medication compliance in older patients living in the community: a systematic review of the literature. Drugs \& Aging, 20(3), 229-240.

Wald, D.S., Bestwick, J.P., Raiman, L., Brendell, R. \& Wald, N.J. (2014) Randomised Trial of Text Messaging on Adherence to Cardiovascular Preventive Treatment (INTERACT Trial). PLOS ONE, 9(12), e114268.

Wilke, T., Müller, S. \& Morisky, D.E. (2011) Toward Identifying the Causes and Combinations of Causes Increasing the Risks of Nonadherence to Medical Regimens: Combined Results of Two German Self-Report Surveys. Value in Health, 14(8), 1092-1100.

World Health Organisation (2004) Atlas of Heart Disease and Stroke. Vol. 2015 WHO, Geneva. 